Biol. Cybern. 61, 71-77 (1989)

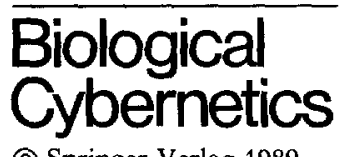

(C) Springer-Verlag 1989

\title{
Control of Body Position of a Stick Insect Standing on Uneven Surfaces
}

\author{
H. Cruse ${ }^{1}$, D. Riemenschneider ${ }^{2}$, and W. Stammer ${ }^{2}$ \\ ${ }^{1}$ Fakultät Biologie der Universität, Postfach 8640, D-4800 Bielefeld 1, Federal Republic of Germany \\ ${ }^{2}$ Fachbereich Biologie der Universität, Postfach 3049, D-6750 Kaiserslautern, Federal Republic of Germany
}

\begin{abstract}
When a multi-legged animal walks over uneven surfaces, each leg has to span a different distance between body and ground. Thus the animal has to solve the problem of how to control the body height, i.e. to coordinate the movement of the legs in such a way that the vertical projections of leg lengths match these distances. For the standing animal, this is investigated here by testing twelve different substrate configurations and measuring body height and forces applied by the legs on the substrate. The results are consistent with the hypothesis that the legs can be considered to represent independent height controllers. They can be understood as proportional controllers with nonlinear characteristics. The mechanical coupling between the leg is sufficient to explain the experimental results. Thus, no neuronal coupling has to be assumed to exist between these controllers. This agrees with a hypothesis proposed earlier for walking animals (Cruse 1976).
\end{abstract}

\section{Introduction}

When an animal walks over an uneven surface the control of the movement of the legs has to cope with two problems: one is the temporal coordination of the different legs. This has been studied intensively (for review, see Graham 1985). The second problem is the control of body height. For insects, only a few investigations consider this question (Wendler 1964; Kemmerling and Varju 1981, 1982; Bässler 1965, 1977; Cruse 1976). All these investigations have been done with the stick insect. The first papers dealt with height control in standing animals and only the latter two considered walking animals. On the basis of experimental results on stick insects walking over obstacles and ditches, one paper (Cruse 1976) concluded with a quite simple hypothesis: it was assumed that each leg acts as an elastic system which controls the distance between body and surface independent of the other legs. This elastic system might be represented simply by the elasticity of the muscles of the leg or it might include the resistance reflex systems found in the standing animal to control the angles of the individual joints (see review Bässler 1983). These systems operate together on the basis of a proportional feedback regulation. Thus according to this hypothesis no neuronal but only mechanical connection was necessary between the legs to perform the height control. The three height controllers - one for each thoracic segment - are described by hypothetical force-height characteristics which can be interpreted as cost or energy functions. Using these terms, the system can be described as adopting that body position which requires the minimum total energy.

In this paper, this hypothesis will be tested for standing animals. To this end, the force-height characteristics will be measured for all legs in the fixed animal and compared with the corresponding characteristic obtained from animals standing free over uneven surfaces. The results show that no essential difference is found between the two measurements, indicating that the characteristic found for the fixed animal are sufficient to describe the behavior of the free standing animal. In the fixed animal, the independence of the individual height controllers will be tested and first investigations will deal with the question as to how body height is measured.

\section{Methods}

Adult female stick insects, Carausius morosus, are used throughout in these experiments. The animals had a mean body weight of $9.1( \pm 1,2) \mathrm{mN}$ (mean \pm S.D.). To measure the force-height characteristics the animals are fixed dorsally to a horizontal holder by means of dental wax (Scutan). The tarsi of the legs are placed on three horizontal balsa beams one for each pair of legs. 
The beams are fixed to force transducers. The orientation of the transducers can be changed to measure different spatial force components. By means of micromanipulators, the transducer and the balsa beam can be moved in a vertical (dorsal-ventral) and horizontal (caudal-rostral) direction. The forces were recorded conventionally on a pen recorder (Hellige $\mathrm{He} 18$, see e.g. Cruse and Saxler 1980). The distance between the beam carrying the tarsi of the front legs and that of the middle legs amounts to $30 \mathrm{~mm}$, the corresponding distance between middle and hind legs to $25 \mathrm{~mm}$. The distal end of the tibia is defined as the position of tarsus.

In the second series of experiments, the animal stands free on the three balsa beams. In addition to force, the height of the body is also measured in the following way. Parallel to the sagittal plane of the animal, a mirror was placed which was covered with a transparent millimeter scale. The height of the upper margin of the thorax directly dorsally to each coxa was read from the millimeter scale after matching the body of the insect with its mirror image to avoid parallax errors. The heights of the three thoracic segments and the forces developed by each pair of legs are measured in 12 different situations: The experimental procedure started in the zero position, where all three beams were on the same, zero level. The middle legs are placed in such a way that the long axis of the femur is oriented horizontally and perpendicular to the long axis of the body. The long axis of the tibia of the middle leg is oriented vertically. After three minutes of zero position, the height of one balsa beam was changed to one of four positions $( \pm 8, \pm 16 \mathrm{~mm})$ using the micromanipulator. The heights and the forces were measured directly after this change (dynamic value) and after 5 min (static value). Then the animal was brought back to zero position. After another $5 \mathrm{~min}$, the next position was investigated. Four height values for the three leg pairs give altogether 12 substrate configurations. The decay of the force values can be roughly approximated by exponential functions (Kemmerling and Varju 1982). The adaptation time of $5 \mathrm{~min}$ was found as a compromise in preliminary experiments. Although adaptation in not quite completed, a longer time would lead to a measurable loss of body height over the whole session which took about $50 \mathrm{~min}$ for the first six substrate configurations. The remaining ones are investigated after giving the animal a two hours rest. Whilst forces show a considerable change during the first $5 \mathrm{~min}$, the dynamic and the static value is presented. The height does not show a significant change in this period. Thus only one height value is given here. To avoid systematic errors due to fatigue, the 12 substrate configurations were chosen in a randomized chronological order.

\section{Results}

\section{Force-Height Characteristics in Fixed Animals}

Ten animals are fixed dorsally and both dynamic and static force-height characteristics are measured for the three pairs of legs - front, middle, and hind legs. In this experimental situation, the weight of the animal is supported by the holder. Therefore not the absolute force value, but the change of force which is obtained when the tarsi are moved up or down is shown for easier comparison. This is possible since according to Kemmerling and Varju (1981) the value of the initial force does not influence the values of the force changes
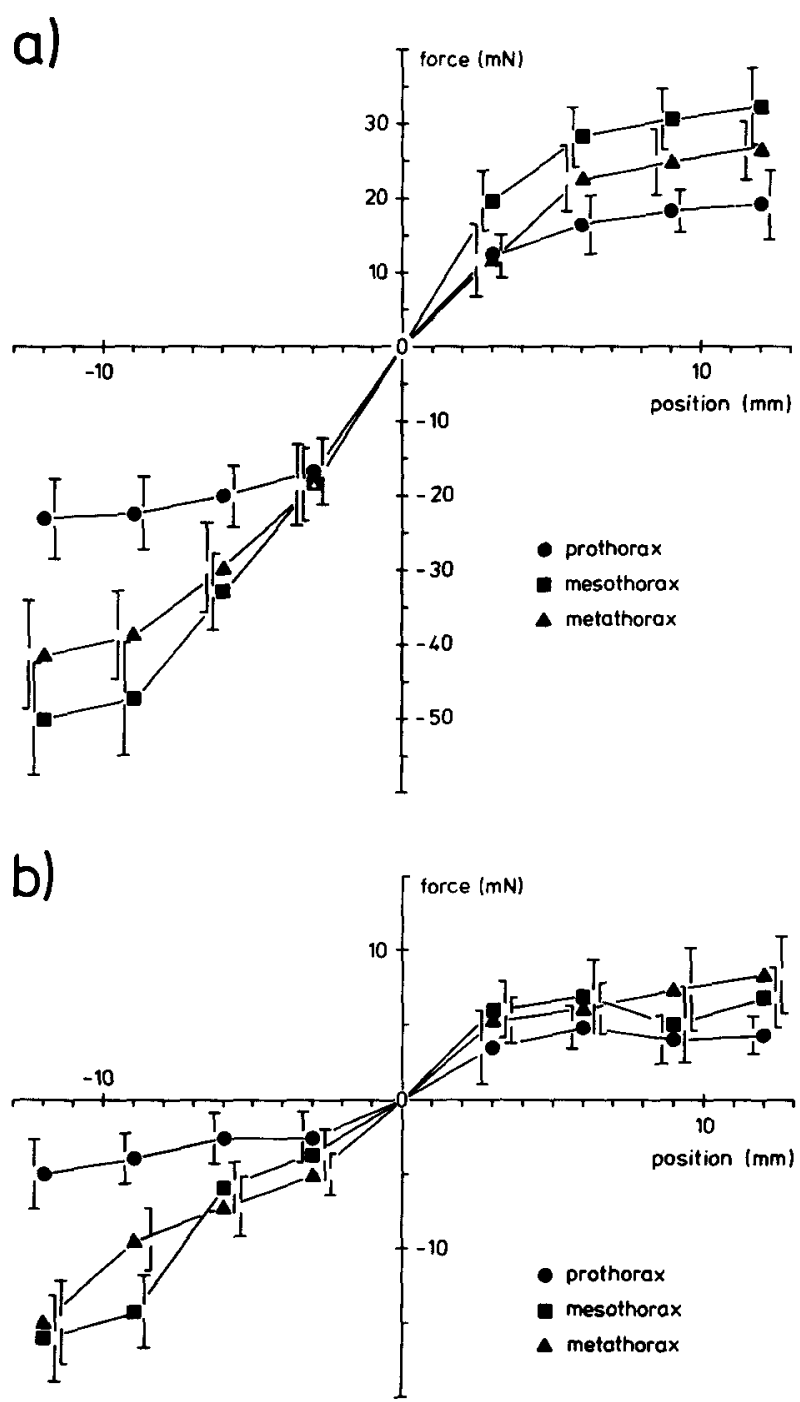

Fig. $1 \mathrm{a}$ and $\mathbf{b}$. Force-height characteristics in fixed animals. The dynamic (a) and static (b) values of the vertical force component for a pair of legs is measured for different vertical displacement of the tarsi. The ordinate shows the change of force values obtained when the tarsus is moved up or down from the zero position. Upward tarsus displacement and downward force is positive. Circles: prothorax, squares: mesothorax, triangles: metathorax. Mean values and standard deviation from 10 animals 
significantly. The movement is always started at the average distance obtained in the zero position of the free standing animal. Only the vertically directed force component is investigated. The results are shown in Fig. 1 for (a) the dynamic and (b) the static case. The characteristics have a nonlinear form. The highest forces are produced by the two middle legs.

Is there an influence from that leg pair which is moved in the experiment onto one of the other two leg pairs which are not moved? The results show that each stimulus (movement of the balsa beam) produces a short force pulse in the other pairs which, however, is less than $2 \mathrm{mN}$. This value is independent of the direction of the stimulus and the corresponding reaction is found when the animal obtained a tactile stimulus anywhere at the body. Electrophysiological investigations showed that a cocontraction of leg muscles occurs in such a situation (Cruse and Schmitz 1983). Thus we conclude that with respect to height control no significant influence can be found between the legs of different segments in the fixed animal.

\section{Force-Height Characteristic}

in the Animal Standing Free on Uneven Surfaces

In a second series of experiments, the stick insect stands free on the three balsa beams. The height of the balsa beams was changed to obtain 12 different substrate configurations (see Methods). As a measure for the height, the upper margin of the three thoracic segments was determined as described in the Methods. The mean body positions are shown graphically in Fig. 2. As can be seen, the spatial orientation of the thoracic segments changes for the different substrate configurations with respect to a spatial, laboratory fixed coordinate system. When the forces measured in the fixed animal are compared with those produced by the free animal, the latter forces have to be known in a body-fixed coordinate system. Because of the different orientation of the body in the free standing case, the horizontal force component also has to be measured. In Fig. 2, the vectorial sum of the two components are shown both for the dynamic (dashed arrows) and for the static case (full arrows).

From these results given in a spatially fixed coordinate system, the thoracic height and the vertical force component, both measured in a body fixed coordinate system, can be calculated. As the orientation of the individual thoracic segments can be somewhat different, this calculation was made separately for each segment and with reference to the orientation of the individual thoracic segment (see Fig. 3c). These values are shown as characteristics in Fig. 3 in the same way as was done for the fixed animal in Fig. 1. The numbers refer to the substrate configurations given in Fig. 2.
(D)

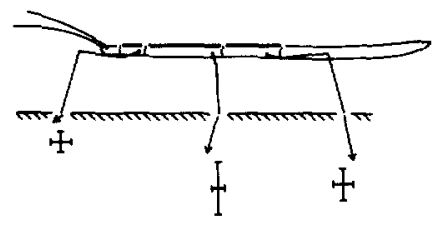

$$
\left[\begin{array}{l}
20 \\
\mathrm{~mm}
\end{array}\right] \begin{aligned}
& 10 \\
& \mathrm{mN}
\end{aligned}\left[\begin{array}{l}
20 \\
\mathrm{mN}
\end{array}\right.
$$

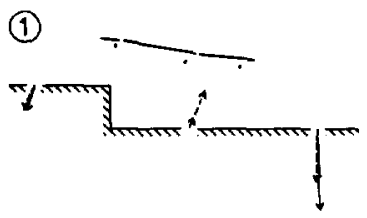

(7)
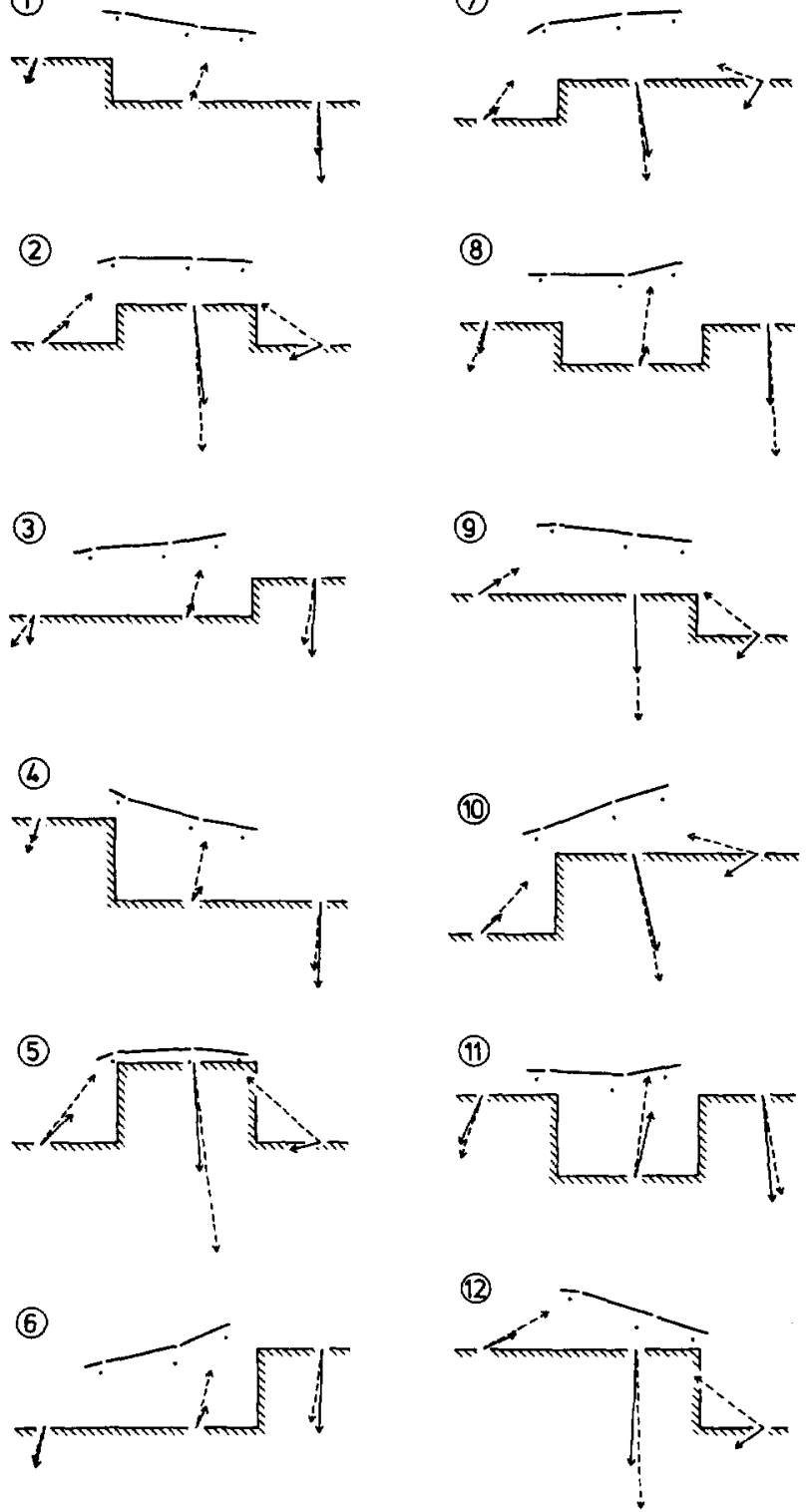

Fig. 2. Free standing animals. Orientation of thoracic segments and forces measured at the tarsi in the vertical plane for 12 different substrate configurations. Bars mark the upper margin of the thoracic segments, dots mark the upper rim of the coxa. Dynamic force values (dashed arrows) are shown using twice the scale factor compared with static force values (full arrows). Values from 10 animals. The uppermost figure, $(0)$, which for illustration includes a schematic sketch of the stick insect shows the zero position and contains the standard deviation of the horizontal and the vertical force components below each of the three leg pairs 

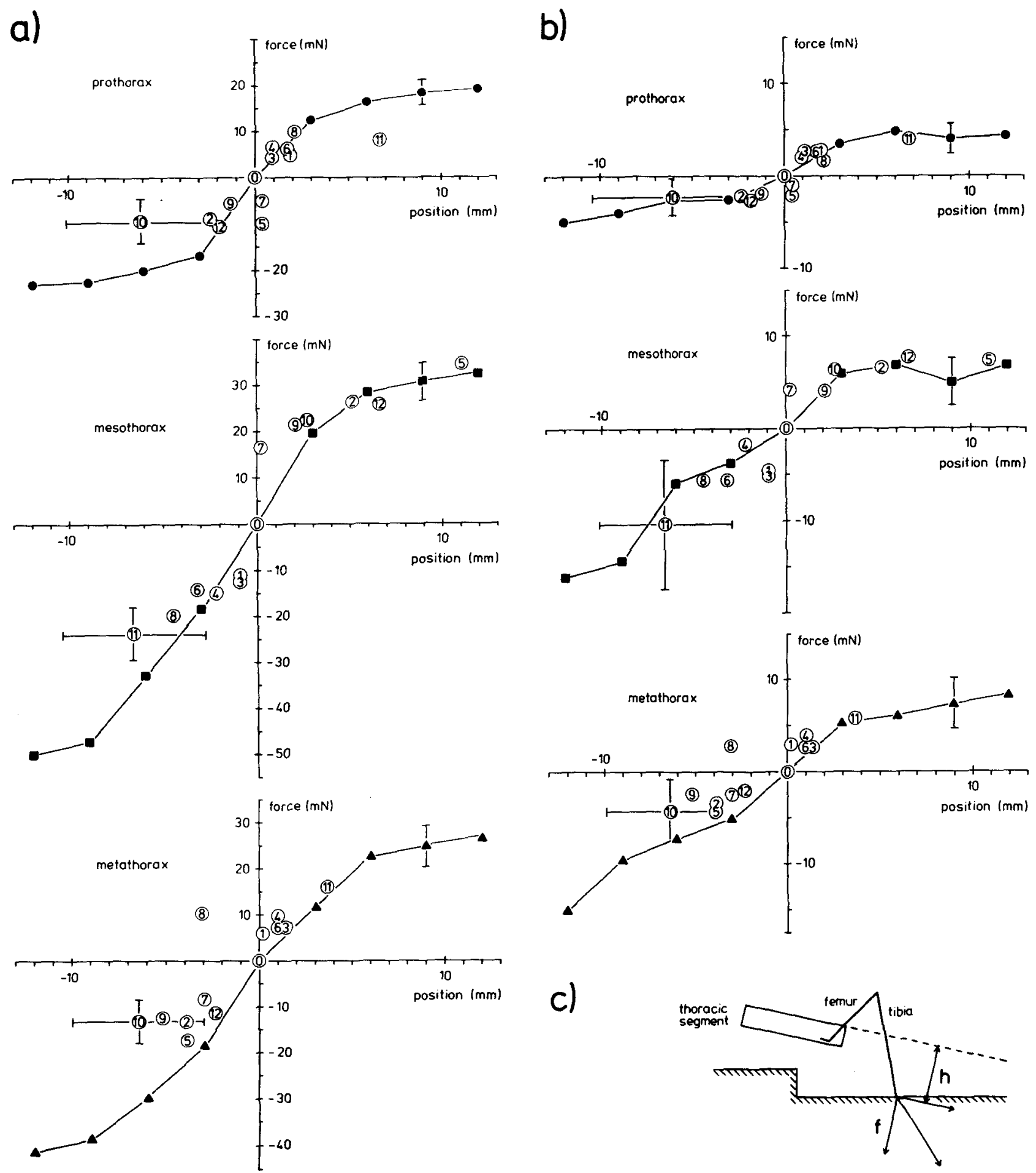

Fig. 3a-c. Force-height characteristics in free standing animals. The dynamic (a) and static (b) values of the force component perpendicular to the long axis of the corresponding thoracic segment versus height measured in the body-fixed coordinate system. In $c$ these values are schematically shown as $f$ and $h$, respectively. In $\mathbf{a}$ and $\mathbf{b}$, as in Fig. 1, the force values obtained in the zero position are defined as zero. The numbers refer to the substrate configurations given in Fig. 2. The vertical and horizontal bars show the corresponding standard deviations which for clarity are only shown for some typical cases. Values from 10 animals. For comparison, the mean values of the characteristics obtained from fixed animals (Fig. 1) are also shown as closed symbols 


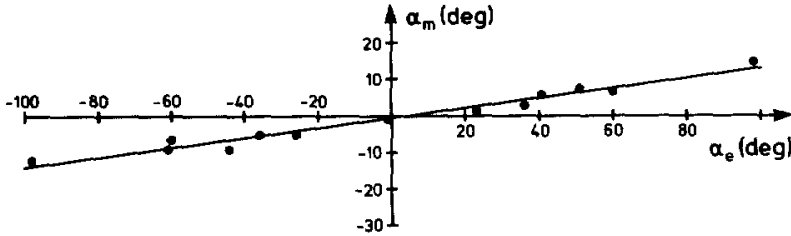

Fig. 4. The values of the measured angle between meso- and metathorax alpha $(m)$ plotted against the values of alpha $(e)$ which would be expected, if the three thoracic segments controlled their height completely independently of one another and the thoracic joints could be moved free

For easier comparison, the force values measured in the fixed animal are again shown as closed symbols in Fig. 3. Taking into account the standard deviations for the height values (horizontal) and the force values (vertical) the results of both experimental series do not show appreciable differences.

As can be seen in Fig. 2, the three thoracic segments are not held completely rigid but change their relative position to a small degree when the form of the surface changes. To obtain a quantitative description of this behavior, the angle between meso- and metathorax was measured and plotted in Fig. 4 versus that hypothetical angle which would have been obtained when the distance between each thoracic segment and its substrate was always constant, i.e. when the thoracic joints were bent so much to completely follow the form of the surface. As shown by Fig. 4, there is a small but highly significant dependence of the angle between meso- and metathorax on the form of the surface which is very similar to that found for free walking animals (Cruse 1976, Fig. 4). The angle between pro- and mesothorax was not measured since a change in this angle does influence the position of the body to an only very small degree because of the very short distance between the coxa of the prothorax and the rostral end of the mesothorax.

In order to be able to compare the results from fixed animals with those of free standing animals, only that force component was considered which runs in the vertical plane and is perpendicular to the long axis of the corresponding thoracic segment. In conjunction with this, only the projection of the height on the direction of this force component was considered (see Fig. $3 \mathrm{c}$ ). However, these parameters might possibly not be those which are the essential parameters, i.e. those which are actually controlled by the nervous system of the animal. In order to find alternative hypotheses concerning these parameters, the resulting forces measured in all 12 substrate configurations are plotted in a body-segment fixed coordinate system (Fig. 5). The forces shown in Fig. 5 represent the vectorial difference between the total force and that force developed by the leg pair in the zero position.
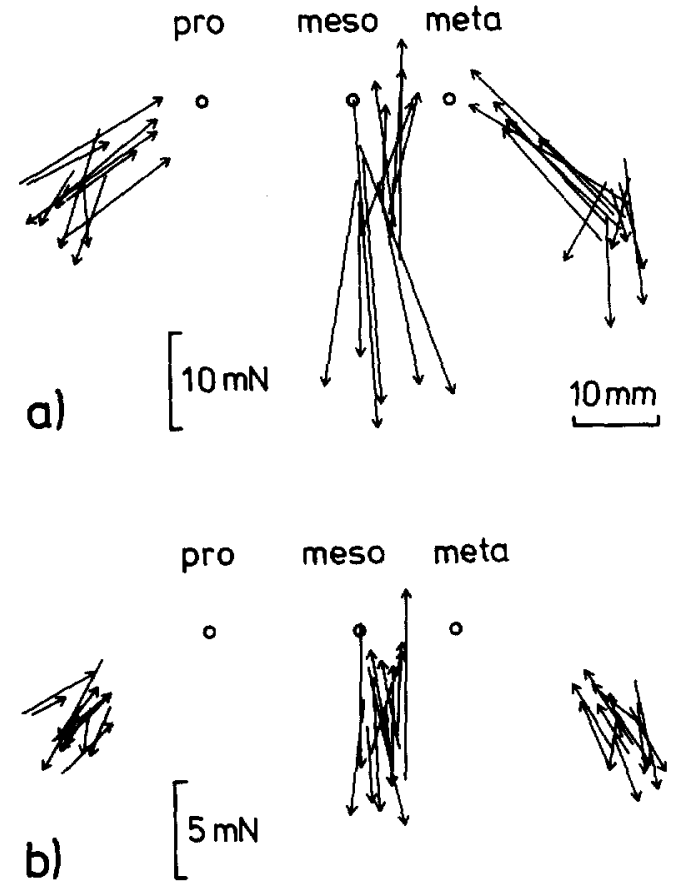

Fig. 5a and b. Free standing animals. The force components which are shown in Fig. 2 in a spatially fixed coordinate system are presented here in body-fixed coordinate systems chosen separately for each thoracic segment. a Dynamic and b static forces. The long axis of the body is assumed to be horizontal. The open circles mark the upper rim of the coxae of pro-, meso-, and metathorax, respectively. In contrast to Fig. 2, not the total force value is shown but only the vectorial difference between the total force and that force which is developed in the zero position. The latter are given in Fig. 2

Thus only the force changes due to the position changes are shown. As can be seen in Fig. 5, all vectors point more or less directly to or directly away from the coxa of the corresponding leg, particularly in the static case (Fig. 5 b). This indicates that the distance between coxa and tarsus might be a better approximation to the essential parameter compared to the vertical height.

This can be investigated by splitting the total force into two perpendicular components, one of which points from tarsus to coxa. When the values of the latter are plotted versus the Euclidean distance between tarsus and coxa, actually a considerable correlation was obtained (not shown). However, the distance between coxa and tarsus cannot be the only parameter as the perpendicular force component seems not to depend on this parameter alone. This can be seen more clearly in the following figure. The "vector field" shown in Fig. 5 for free standing animals can also be investigated in the fixed animal. However, as was shown in Fig. 3, a much larger range of leg positions can be measured in the latter case. The results from such measurements using the hind leg as an example are 

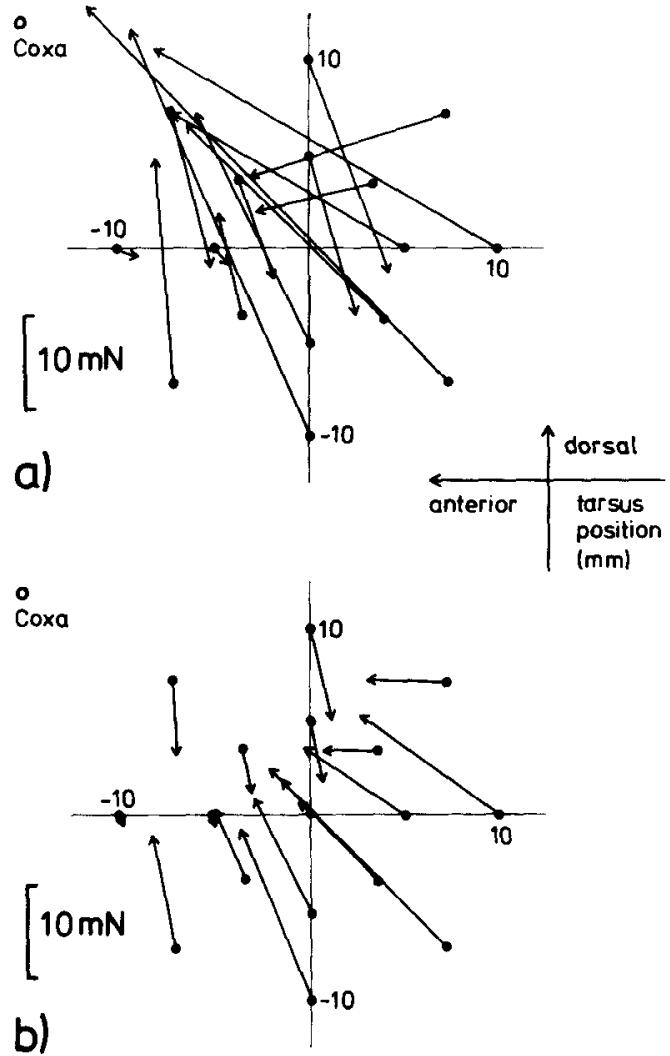

Fig. 6a and b. Fixed animals, hind leg. Dynamic (a) and static (b) force vectors in the vertical plane for different positions of the hind leg tarsus (dots). Coordinate system as in Fig. 5. The origin corresponds to the zero position. For the dynamic forces the mean standard deviation is $\pm 6.6 \mathrm{mN}$, the highest value is $\pm 14.6 \mathrm{mN}$, the lowest $\pm 1.4 \mathrm{mN}$. For the static forces, the corresponding values are $\pm 4.3 \mathrm{mN}, \pm 12.3 \mathrm{mN}$, and $\pm 1.2 \mathrm{mN}$, respectively. Values from 10 animals

shown in Fig. 6. Comparison of Fig. 5, hind legs, and Fig. 6 shows that in the overlapping range these vector fields match considerably. However, no immediately obvious geometric parameters are found which could be easily correlated with the force values.

\section{Discussion}

The results on free standing animals agree with the hypothesis that the position of the body is produced by three independent height controllers which are only coupled mechanically. The characteristics found for the free standing animal show no significant difference from the corresponding force-height characteristics of the fixed animal (Fig. 3). The same correspondence was found for the vector field in the hind leg (Figs. 5 and 6). It could be shown for fixed animals (Cruse and Schmitz, in preparation) that the force-height characteristics of a single leg have half the force value of the characteristics found here for a pair of legs. Thus one can assume that not only the "height controller" of a segment, but even those of the individual legs act independently of each other. Thus the hypothesis proposed for the control of body height in walking animals (Cruse 1976) can also be applied to free standing animals. Furthermore, the weak "elasticity" in the meso-metathoracic joint was also found to be quite similar in both free standing and walking animals. The slopes of the regression lines are 0.136 and 0.082 , respectively.

However, the question as to the parameters which are controlled by these "height" controllers still remains open. Each leg produces a force in the zero position, i.e. when all legs stand on a horizontal plane. The results show that any deviation from this zero position leads to a change of the total force vector. The direction and the amount of this deviation influences the direction and magnitude of the vectors describing the change of force. This can be demonstrated in form of a vector field as is shown in Fig. 5 for all legs of the free standing animal and in Fig. 6 for the hind legs of the fixed animal. The arrangement of these vectors could provide hints on the kind of the parameters on which these force changes depend. Because all vectors roughly point to or away from the coxa in Fig. 6, as a first and rough approximation this vector field might be considered as to be based on a polar coordinate system the origin of which is the coxa of the leg. However, closer inspection immediately shows obvious deviations from such a simple topological arrangement.

Since the forces result from the torques produced in the different joints of the leg, the following assumption might be the easiest explanation for these distortions. The control systems of each joint might act as independent proportional controllers and the topological arrangement of the force vectors might simply result from the spatial summation of the torques which are produced independently in each joint. Calculation of these torques would require measurement of all three spatial force components instead of only two as was done here. Therefore investigations on whether the force vectors can directly be traced back on the properties of the controllers of the individual joints has to be reported in a forthcoming paper.

\section{References}

Bässler U (1965) Proprioreceptoren am Subcoxal- und FemurTibia-Gelenk der Stabheuschrecke und ihre Rolle bei der Wahrnehmung der Schwerkraftrichtung. Kybernetik 2:168-193

Bässler U (1977) Sensory control of leg movement in the stick insect Carausius morosus. Biol Cybern 25:61-72 
Bässler U (1983) Neural basis of elementary behavior in stick insects. Springer, Berlin Heidelberg New York

Cruse H(1976) The control of the body position in the stick insect (Carausius morosus), when walking over uneven surfaces. Biol Cybern 24:25-33

Cruse H, Saxler G (1980) Oscillations of force in the standing legs of a walking insect (Carausius morosus). Biol Cybern 36:159-163

Cruse H, Schmitz J (1983) The control system of the femur-tibia joint in the standing leg of a walking stick insect Carausius morosus. J Exp Biol 102:175-185

Graham D (1985) Pattern and control of walking in insects. Adv Insect Physiol 18:31-140

Kemmerling S, Varju D (1981) Regulation of the body-substratedistance in the stick insect: responses to sinusoidal stimulation. Biol Cybern 39:129-137
Kemmerling S, Varju D (1982) Regulation of the body-substratedistance in the stick insect: step responses and modelling the control system. Biol Cybern 44:59-66

Wendler G (1964) Laufen und Stehen der Stabheuschrecke: Sinnesborsten in den Beingelenken als Glieder von Regelkreisen. Z Vergl Physiol 48:198-250

Received: December 16, 1988

Accepted: January 18, 1989

Professor Dr. Holk Cruse

Fakultät Biologie der Universität

Postfach 8640

D-4800 Bielefeld 1

Federal Republic of Germany 